\title{
Air pollution as a contributor to the inflammatory activity of multiple sclerosis
}

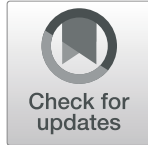

\author{
Andrea Cortese ${ }^{1,2^{*}} \mathbb{D}$, Luca Lova ${ }^{3 \dagger}$, Patrizia Comoli ${ }^{4 \dagger}$, Elisabetta Volpe ${ }^{5}$, Silvia Villa ${ }^{6}$, Giulia Mallucci ${ }^{1,6^{*}}$, \\ Sabrina La Salvia ${ }^{6}$, Alfredo Romani ${ }^{6}$, Diego Franciotta ${ }^{6}$, Valentina Bollati ${ }^{7}$, Sabrina Basso ${ }^{4}$, Ilaria Guido ${ }^{4}$, \\ Giuseppe Quartuccio ${ }^{4}$, Luca Battistini ${ }^{5}$, Cristina Cereda ${ }^{6}$ and Roberto Bergamaschi ${ }^{6^{*}}$
}

\begin{abstract}
Objective: Air pollution has been recently identified as a risk factor for multiple sclerosis. Aim of this study was to investigate the immunological mechanism underlying the clinical association between air pollution, namely exposure to particulate matter 10 (PM10), and inflammatory activity of multiple sclerosis (MS)
\end{abstract}

Methods: Daily recording of PM10 was obtained by monitors depending on the residence of subjects. Expression of molecules involved in activation, adhesion, and migration of T lymphocytes were tested by flow cytometry in 57 MS patients and 19 healthy controls. We next assessed in vitro the effect of PM10 on expression of C-C chemokine receptors 6 (CCR6) by peripheral blood mononuclear cells (PBMCs), on cytokine production by monocyte-derived dendritic cells (mdDC), and on T cell polarization in PBMC/mdDC mixed cultures.

Results: We identified a significant correlation between mean PM10 levels and expression of CCR6 CD4+ T circulating cells in MS patients. This was paralleled by the observation in vitro of a higher level of CCR6 expression on PBMC following treatment with increased doses of particulate matter. Moreover, in mdDC cultures, particulate matter induced the secretion by mdDC of Th17 polarizing IL1 beta, IL6, and IL23 and, in mdDC/PBMC mixed cultures, enhanced generation of IL17-producing T cells.

Conclusions: Ex vivo and in vitro studies support the pro-inflammatory role of PM in MS, by upregulating expression of CCR6 on circulating CD4+ T cells and inducing in innate immune cells the production of Th17 polarizing cytokines. Therefore, we speculate that in MS respiratory exposure to PM10 may induce the production in the lung of autoreactive Th17 lymphocytes and boost their migratory properties through the blood-brain barrier.

Keywords: Air pollution, Particulate matter, Multiple sclerosis, Th 17 lymphocytes, Adhesion molecules

\section{Introduction}

Multiple sclerosis (MS) is an inflammatory demyelinating disorder of the central nervous system (CNS) which is characterized in its early phase by periods of exacerbation and remission along with progressive neurologic disability. During MS relapses, inflammatory cells

\footnotetext{
*Correspondence: andrea.cortese@unipv.it; giulia.mallucci@mondino.it; roberto.bergamaschi@mondino.it

${ }^{+}$Luca Lova and Patrizia Comoli contributed equally to this work.

${ }^{1}$ Department of Brain and Behavioural Sciences, University of Pavia, Pavia, Italy

${ }^{6}$ IRCCS Mondino Foundation, Pavia, Italy

Full list of author information is available at the end of the article
}

migrate through the blood-brain barrier (BBB) inside the CNS, where autoreactive $T$ cells are reactivated, leading to local inflammatory demyelination, disruption of the $\mathrm{BBB}$, and subsequent enhanced extravasation of leukocyte $[1,2]$.

MS relapse occurrence varies during the year, and different studies reported an increased rela pse rate in either autumn/winter or spring/summer [3]. Infections, low vitamin $\mathrm{D}$, and low melatonin levels have been associated to increase relapse occurrence $[4,5]$. Moreover, dietary habits, by modifying the gut microbiota [6,7], stress, and smoking have also been linked to increase incidence and MS exacerbation [8]. However, none of these factors can

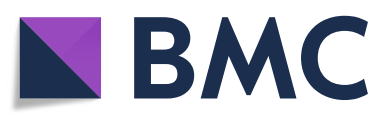

(c) The Author(s). 2020 Open Access This article is licensed under a Creative Commons Attribution 4.0 International License, which permits use, sharing, adaptation, distribution and reproduction in any medium or format, as long as you give appropriate credit to the original author(s) and the source, provide a link to the Creative Commons licence, and indicate if changes were made. The images or other third party material in this article are included in the article's Creative Commons licence, unless indicated otherwise in a credit line to the material. If material is not included in the article's Creative Commons licence and your intended use is not permitted by statutory regulation or exceeds the permitted use, you will need to obtain permission directly from the copyright holder. To view a copy of this licence, visit http://creativecommons.org/licenses/by/4.0/. The Creative Commons Public Domain Dedication waiver (http://creativecommons.org/publicdomain/zero/1.0/) applies to the data made available in this article, unless otherwise stated in a credit line to the data. 
fully explain the variability of MS course, thus suggesting that additional factors may play a role.

In a recent study, we have established a significant association between air-borne particulate matter levels and inflammatory activity of the disease, as expressed by the presence of gadolinium-enhancing lesions on brain MRI in MS patients [9]. Other groups have also observed an association between ambient levels of air pollution and MS prevalence [10], as well as clinical activity of the disease [11-14].

Altogether, these observations support a role for air pollution as a novel risk factor for MS, favoring its inflammatory exacerbations. However, the mechanisms underlying this clinical observation remain unknown.

Therefore, here, we investigated by the effect of the exposition to particulate matter 10 (PM10) on inflammatory markers on circulating CD4 and CD8 lymphocytes in MS patients and controls. As an association between PM10 and the expression of the migration marker CCR6 was identified, we performed in vitro studies in order to further confirm the effect of PM10 on its expression, as well as on cytokine production and $\mathrm{T}$ cell polarization.

\section{Methods}

\section{Subjects}

Venous blood samples were obtained between December 2013 and April 2015 from 57 prospectively enrolled relapsing remitting (RR) MS patients with clinically and radiologically stable disease and 19 healthy controls, who were recruited among people having the same residence of enrolled MS patients. Mean age of patients was $43 \pm$ 10 years, 24 (42\%) were males, 15 (26\%) were smoker, 31 (54\%) were on disease modifying treatment. Mean EDSS was $1.4 \pm 1.5$ (range 0-6), and mean disease duration was $7.8 \pm 9$ years. Mean age of controls was $46 \pm 12$ years; 10 (53\%) were males; 5 (26\%) were smoker.

The Ethics Committee of San Raffaele Hospital, Milan, Italy, approved study protocol, and all subjects signed informed consent form.

\section{Air pollution measurement}

Ambient concentrations of PM10, which is physically defined by the mass median aerodynamic diameter < $10 \mu \mathrm{m}$ of the pollutant particles, were obtained from the regional air-quality monitoring network (ARPA, Regional Environmental Protection Agency). PM10 concentrations were obtained from the seven stations located in the Pavia province, as all the study participants were resident in that area.

The addresses of monitoring stations and study subjects were geocoded in order to assign to each subject the daily PM10 concentration from the nearest monitor to home address. None of the cases enrolled reported the use of home air filters. For patients who worked far from their residence, mean daily PM10 levels at residence and work place were averaged. PM10 exposure was considered from the date of blood draw to the 15 days before. The 15 days' time period was chosen based on our previous work showing an association between the presence of gadolinium-enhancing lesion on brain MRI and the level, which was higher at 15 days prior to the MRI scan [9]. Therefore, mean PM10 level 15 days prior to blood draw was also used in the models of this study.

\section{Flow cytometry}

Blood samples were freshly analyzed by lyse-and-wash whole blood staining procedure. Surface staining was performed on ice for $20 \mathrm{~min}$, and the cells were then analyzed on a two laser, six color FACSCanto flow cytometer. Multiplexed dilutions of monoclonal antibodies (mAbs) were used to characterize lymphocyte populations. The following antibodies from $\mathrm{BD}$ Biosciences were used: CD69-FITC, HLA-DR-FITC, CD49d-PE, CD11a-PE-Cy7, CD4APC-H7, CD8 APC-H7, CCR6PerCP-Cy5.5, CD183 PE-Cy 7, CD44- PerCP-Cy 5.5.

\section{PBMC and mixed mdDC/PBMC cultures}

PBMCs were separated by Ficoll-Hypaque centrifugation (Amersham Biosciences) from buffy coats of healthy blood donor volunteers and were cultured according to standard procedures in presence of different doses of particulate matter (1648a, urban particulate matter, National Institute of Reference Material, USA; final concentration used after optimisation $5-20 \mu \mathrm{g} / \mathrm{ml}$ for PBMC and $10-40 \mu \mathrm{g} / \mathrm{ml}$ for $\mathrm{mdDC}$ and $\mathrm{mdDC} / \mathrm{PBMC}$ cocultures). mdDC were generated from peripheral blood monocytes as described previously [15]. For mixed cultures, PBMC were cocultured with unmanipulated mdDC, or with mdDC pulsed with PM10 (PBMC:mdDC ratio of 100:1), for 8 days in RPMI medium supplemented with $10 \%$ fetal calf serum (Hyclone). Cytokine secretion profile was evaluated on culture supernatant by ELISA and on cultured cells by ELISPOT assay.

\section{ELISPOT and ELISA assays}

ELISPOT assays were performed according to a previously described method [15]. Briefly, 96-well multiscreen filter plates (MAIPS 4510, Millipore, Bedford, MA) were coated with $100 \mu \mathrm{l}$ of primary antibody (IL17; Mabtech, Nacka, Sweden) at $2.5 \mu \mathrm{g} / \mathrm{ml}$, and incubated overnight at $4{ }^{\circ} \mathrm{C}$. Cultured cells were plated at $1 \times 105$ cells/well in triplicate. After incubation for $24 \mathrm{~h}$ at $37^{\circ} \mathrm{C}, 100 \mu \mathrm{l}$ of biotinylated secondary antibody (Mabtech, $0.5 \mu \mathrm{g} / \mathrm{ml}$ ) was added, and plates were then processed according to standard procedure. Cytokine-producing spots were counted using an ELISPOT reader (Bioline, Torino, Italy). The number of spots per well was calculated after 
subtracting the assay background, quantitated as an average of 24 wells containing only sterile complete medium, and specific background, quantitated as the sum of cytokine spots associated with responders alone.

Cytokine levels in the supernatant of DCs or DCPBMC cocultures were measured using monoclonal antibody pairs (IL1 beta and IL17: Mabtech; IL6: Endogen Tema, Castenaso, Italia; IL23: U-Cytech Biosciences, Utrecht, The Netherlands). Plates were coated with purified antibodies at the appropriate concentrations. Standard curves were prepared with recombinant human cytokines. Biotin-labeled antibodies were added and HRP-conjugated streptavidine was used to develop the reactions. Plates were read at $450 \mathrm{~nm}$ (Titertek Plus MS $212 \mathrm{M})$. Results were reported as picograms per milliliter.

\section{Statistical analyses}

The sample was described by means of mean and standard deviation for continuous variables and proportions for categorical ones. Spearman's coefficient was used to test the correlation between mean fluorescence intensity for the molecules tested and PM10 levels. Association between correlated molecules and PM10 was further tested in a multivariate linear regression model including age, gender, treatment with disease-modifier drugs (yes/ no), and smoker status. Continuous variables compared different PM exposure conditions tested in vitro using non-parametric or parametric two-tailed paired or unpaired Student's $t$ test. Statistical significance was taken at the $<0.05$ level. All analyses were conducted using STATA/SE for Windows, version 12.1 (StataCorp, College Station, TX, USA). Graphs were generated using the GraphPad Prism v7 Software (San Diego, CA, USA).

\section{Results}

In MS patients, PM10 boosts the expression of CCR6 on circulating lymphocytes

In MS patients, but not in controls, we observed a significant correlation between mean PM10 level in the previous 15 days and expression on $\mathrm{CD} 4+\mathrm{T}$ cells of CCR6, a chemokine receptor characteristic of Th17 cells (Fig. 1 and Tables 1 and 2).

In a linear regression model, the association between exposure to PM10 and the expression of CCR6 $(\beta=7.8$, $95 \% \mathrm{CI}=3.2-12.5, p<0.001)$ on $\mathrm{CD} 4+\mathrm{T}$ cells was independent from age, sex, treatment, and smoker status (Table 3). A weak correlation was also observed between PM10 levels and expression of LFA1 on CD4+ T cells, which was not confirmed in an age-, sex-, treatmentand smoker status-adjusted regression model.

Patients were followed up after 3 months, but none of them had a clinical relapse, thus preventing further analysis of the correlation between expression of activation

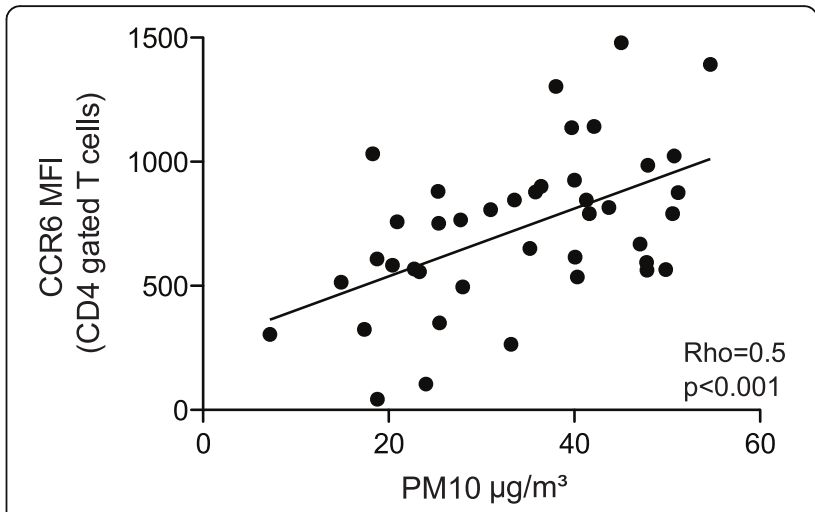

Fig. 1 Scatterplot of expression of CCR6 on CD4+ T cells in MS patients. CCR6: chemokine receptor 6; MFI: mean

fluorescence intensity

and adhesion molecules and clinical activity of the disease in our study population.

The association between CCR6 expression on CD4+ T cells, marker characteristic of the Th17 lineage, and PM10 may suggest a prominent effect of PM10 exposure on Th17-driven inflammatory response.

\section{In vitro PM10 induces expression of migratory markers} on PBMCs and enhance DC-dependent generation of IL17-producing T cells

We next tested in vitro the effect of urban PM on expression of CCR6 as well as on cytokine production and $\mathrm{CD} 4+\mathrm{T}$ cell polarization. PM10 increased the expression of CCR6 on CD4+ T cells (Fig. 2a). Based on the hypothesis that the effect of PM10 on amplification of CCR6+ Th17 cells may be mediated by innate immunity cells residing in the lung where they may uptake airborn PM, we co-cultured PBMCs with PM-treated mdDC and analyzed IL1 beta, IL6, and IL23 cytokine production by $\mathrm{mdDC}$ and assessed in $\mathrm{mdDC} / \mathrm{PBMCs}$ mixed cultures the effect of PM treatment on Th17 cell polarization. In vitro treatment of $\mathrm{mdDC}$ with $\mathrm{PM}$, even at low concentration, induced a massive release of all Th17-polarizing cytokines tested, IL1 beta, IL6, and IL23 (Fig. 2b). As well known, these cytokines are necessary for differentiation of Th17 into fully pathogenic cells $[16,17]$. Co-culture of PBMCs with PM-treated mdDC significantly increased the number of IL17-producting $\mathrm{T}$ cells on ELISPOT-based assay and increased IL17 cytokine level in cell culture medium (Fig. 2c).

These findings support both presence of a direct effect of PM10 on expression of CCRs on CD4+ T cells and a predominantly indirect effect of PM10 on Th17 polarization, by increasing the production of Th17 polarizing cytokines by dendritic cells. 
Table 1 Mean and standard error of expression level, here indicated as mean fluorescence intensity (MFI), of the tested molecules on CD4+ and CD8+ in MS patients and healthy controls

\begin{tabular}{|c|c|c|c|c|}
\hline & \multicolumn{2}{|l|}{ CD4 T cells } & \multicolumn{2}{|l|}{ CD8 T cells } \\
\hline & MS patients $(n=57)$ & Healthy controls $(n=19)$ & MS patients $(n=57)$ & Healthy controls $(n=19)$ \\
\hline \multicolumn{5}{|c|}{ Activation markers } \\
\hline CD69 & $224 \pm 7(n=31)$ & $205 \pm 8(n=9)$ & $280 \pm 10(n=30)$ & $285 \pm 13(n=9)$ \\
\hline HLA DR3 & $1008 \pm 93(n=21)$ & $1376 \pm 392(n=8)$ & $1420 \pm 139$ & $1406 \pm 380(n=8)$ \\
\hline CD38 & $586 \pm 88(n=21)$ & $476 \pm 94(n=8)$ & $735 \pm 92$ & $519 \pm 110(n=8)$ \\
\hline \multicolumn{5}{|c|}{ Adhesion and migration markers } \\
\hline CD44 & $31,582 \pm 1321$ & $23,702 \pm 3122$ & $26,553 \pm 1229$ & $17,792 \pm 2288$ \\
\hline VLA4 & $2300 \pm 85$ & $2518 \pm 185$ & $3102 \pm 108$ & $3214 \pm 161$ \\
\hline LFA1 & $6073 \pm 242$ & $7140 \pm 561$ & $10594 \pm 373$ & $12436 \pm 1041$ \\
\hline CCR6 & $739 \pm 42(n=55)$ & $808 \pm 72(n=18)$ & $478 \pm 28(n=55)$ & $575 \pm 52(n=18)$ \\
\hline CXCR3 & $667 \pm 40$ & $861 \pm 111$ & $815 \pm 59$ & $736 \pm 97$ \\
\hline
\end{tabular}

\section{Discussion}

Incidence of autoimmune disorders has steadily increased in the past century in industrialized countries, thus suggesting that a change in their environment may underlie this epidemiological observation.

Air pollution is increasingly recognized as a major public health concern, and an increased concentration of airborne PM, one of the most studied component of air pollution, has been associated with both increased morbidity and mortality [18].

PM is a complex mixture of constituent chemicals. It is often classified by particle size, although this physical classification may oversimplify the molecular makeup, which may vary from urban to rural areas, including elemental and organic carbons, metals, sulfates, nitrates, and microbial contaminants [19].

Previous studies by us and others found an association between concentration of PM10 in the weeks preceding MS inflammatory activity, demonstrated both by clinical exacerbations [11] and by detection of contrastenhancing lesions on brain MRI [9].

The findings of the present research suggest that air pollution may lead to MS inflammation by two different mechanisms: (1) PM10 may directly lead to upregulation of CCR6 on circulating lymphocytes, which may facilitate their entry to the CNS; (2) PM10 could increase IL1 beta, IL6, and IL23 production by DC and enhance DCdependent generation of IL17-producing T cells.

Migration of pathogenic self-reactive $\mathrm{T}$ cells to the CNS is an essential step for the development of EAE in immunized mice [20]. Migration of lymphocytes through the $\mathrm{BBB}$ is strictly controlled and requires the sequential interaction of AMs on T cells and BBB endothelial cells. In particular, CCR6 is known to mediate early recruitment of Th17 lymphocytes to CNS through its interaction with CCL20 (Chemokine Ligand 20) on choroid plexus and mice lacking CCR6 were shown to be highly resistant to the induction of EAE [21], and increasing

Table 2 Correlation between average PM10 levels 15 days before enrolment and expression level of the all tested molecules on CD4+ and CD8+

\begin{tabular}{|c|c|c|c|c|}
\hline \multirow{2}{*}{$\begin{array}{l}\text { Spearman's Rho between mean of } \\
\text { PM10 } 15 \text { days before enrolment and: }\end{array}$} & \multicolumn{2}{|c|}{ Healthy Controls $(n=19)$} & \multicolumn{2}{|c|}{ MS patients $(n=57)$} \\
\hline & CD4 T cells & CD8 T cells & CD4 T cells & CD8 T cells \\
\hline \multicolumn{5}{|l|}{ Activation markers } \\
\hline CD69 & $-0.2, p=0.58$ & $0.22, p=0.55$ & $0.08, p=0.66$ & $-0.07, p=0.70$ \\
\hline HLA DR3 & $0.22, p=0.60$ & $0.35, p=0.40$ & $-0.14, p=0.53$ & $-0.30, p=0.18$ \\
\hline CD38 & $0.31, p=0.45$ & $0.36, p=0.38$ & $0.004, p=0.99$ & $0.01, p=0.96$ \\
\hline \multicolumn{5}{|l|}{ Adhesion and migration markers } \\
\hline CD44 & $0.13, p=0.6$ & $0.45, p=0.06$ & $0.22, p=0.1$ & $0.23, p=0.08$ \\
\hline VLA4 & $-0.05, p=0.84$ & $0.13, p=0.6$ & $0.17, p=0.21$ & $0.12, p=0.36$ \\
\hline LFA1 & $0.02, p=0.92$ & $0.17, p=0.5$ & $0.26, p=0.05$ & $-0.11, p=0.44$ \\
\hline CCR6 & $-0.02, p=0.93$ & $-0.22, p=0.37$ & $0.43, p=0.001$ & $0.23, p=0.09$ \\
\hline CXCR3 & $-0.02, p=0.94$ & $-0.01, p=0.96$ & $0.10, p=0.44$ & $0.03, p=0.83$ \\
\hline
\end{tabular}

Values indicated are Spearman's Rho coefficient, $p$ value 
Table 3 Multivariate analysis showing the association of CCR6 expression on CD4+ T cells and PM10 level

\begin{tabular}{lll}
\hline & Coeff. [95\% Conf. interval] & $\boldsymbol{p}>|\boldsymbol{t}|$ \\
\hline Mean PM10 level & $\mathbf{5 . 7}[\mathbf{1 . 8 - 9 . 7}]$ & $\boldsymbol{p}=\mathbf{0 . 0 0 5}$ \\
Age & $-4.8[-11.5-1.81$ & $p=0.15$ \\
Male Gender & $-22.4[-164.2-119.3]$ & $p=0.75$ \\
Disease modifying therapy & $-38.3[-187.8-111.1]$ & $p=0.6$ \\
Smoker & $-105.6[-263.2-51.9]$ & $p=0.18$ \\
\hline
\end{tabular}

evidence support a crucial role of Th17 in the pathogenesis of EAE and as auto-aggressive cells in MS [22-24].

Although we did not observe a similar correlation between CCR6 expression and mean PM10 exposure in 19 healthy controls, we acknowledge that a larger number of healthy individuals would need to be tested before drawing firm conclusions on a possible MS-specific effect of PM10.

Our work suggests that PM can boost Th17 polarization by a DC-dependent IL1 beta, IL6, and IL23 release. To this regard, IL17 was also significantly increased in lung or intestine of rats after experimental PM inhalation or ingestion, respectively $[25,26]$. PM10

a

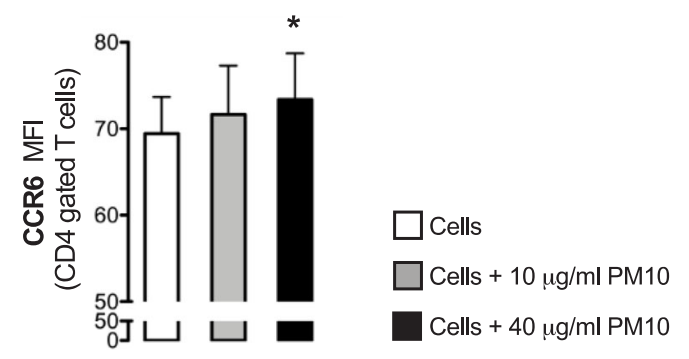

b
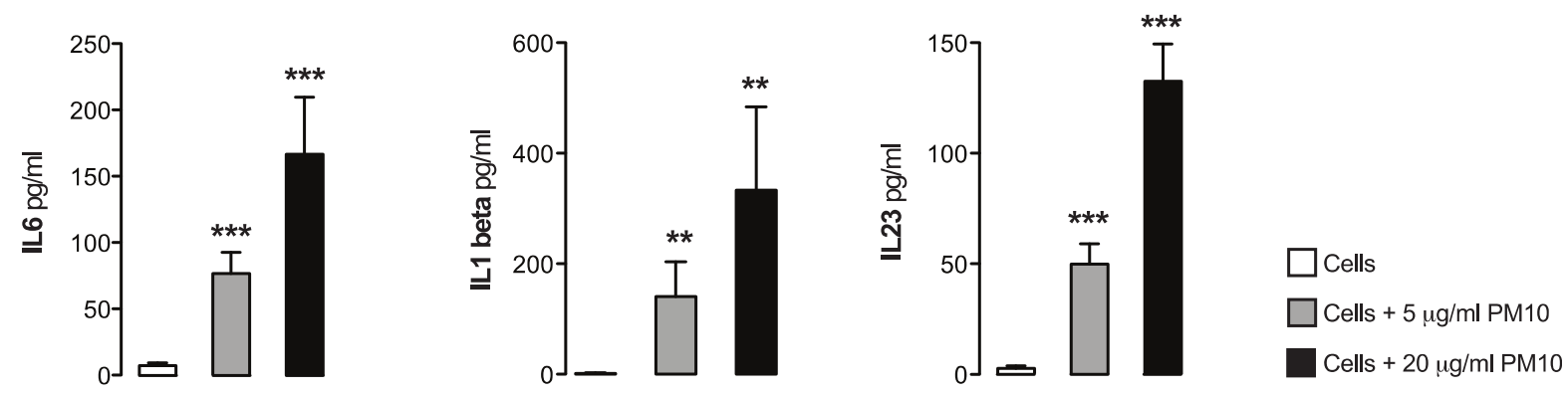

C
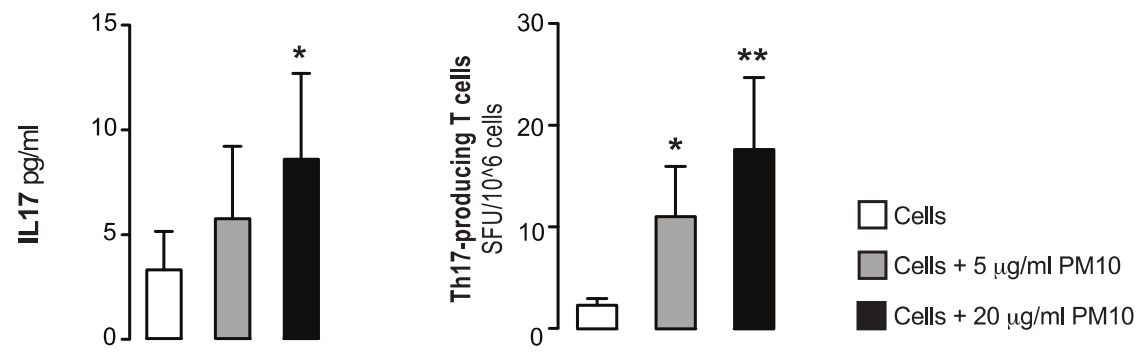

Fig. 2 In vitro treatment of PMBCs with PM induces CCR6 expression on CD4+ cells (a). In vitro treatment of mdDCs with increasing doses of PM10 induces secretion of IL-6, IL1 beta, and IL-23 (b). In mixed mdDC-PBMC co-cultures, in vitro treatment of mdDC PM induces release of IL17and increases the percentage of IL-17 producing T cells (c). ${ }^{*} p<0.05,{ }^{* *} p<0.001,{ }^{* * *} p<0.0001$ 
is known to contain aryl hydrocarbon receptor ligands, and their interaction with the aryl hydrocarbon receptor, which has been shown to play a role in Th17 differentiation and IL17 secretion [27], could represent a possible mechanism of its activity on the immune response.

The present results also imply a crucial role of DC in inflammatory response to PM10. Of note, previous studies similarly found that in vitro urban PM enhanced DC maturation and IL6 production [26, 28]. Importantly, diesel dust, which is over $90 \%$ elemental carbon, did not induce their release, thus suggesting that the cytokineinducing activity of PM10 could be ascribed to different chemical composition of PM compared to diesel dust, including a higher content of extractable organic carbon and inorganic constituents such as sulfates, nitrates, and metals, as well as microbial component. Interestingly enough, in a previous study, pre-treatment of urban air particles with polimixin B and heat shock inactivated its pro-inflammatory effect, thus pointing to a major role of microbial contaminants in proinflammatory effect of PM10 [26].

Altogether, these observations led us to hypothesize that lung resident DC may uptake PM10 in the lower respiratory tract and, after migration to bronchial associated lymphoid tissue, induce a proinflammatory

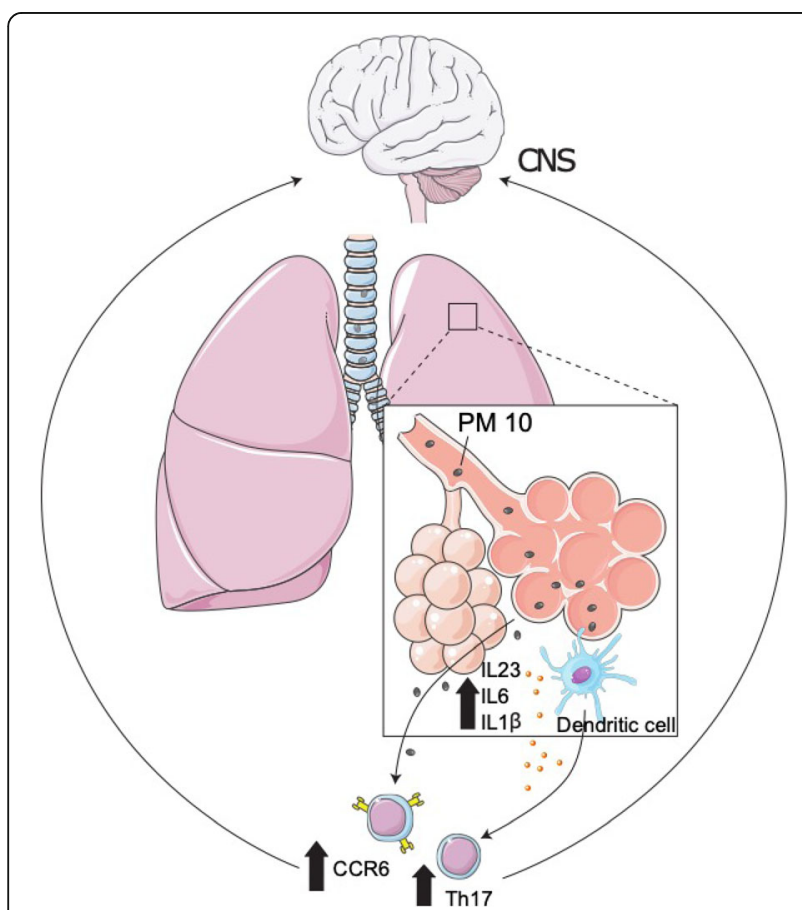

Fig. 3 Respiratory exposure to PM10 may lead to increase disease activity in MS patients by inducing a direct upregulation of adhesion molecules and chemokine receptors on circulating lymphocytes and by promoting lung-resident dendritic cell-mediated release of IL1 beta, IL6, and IL23 and subsequent enhanced generation of Th17producing $\mathrm{T}$ cells response with generation of Th17 cells and enhance their migratory properties, ultimately leading to clinical exacerbation of the disease (Fig. 3). This observation parallels previous evidence in the experimental autoimmune encephalomyelitis, the animal model of MS, of a primary role of the lung in licensing auto-reactive lymphocytes to enter CNS by boosting their migratory properties [29].

\section{Conclusions}

The study shows a pro-inflammatory role of PM in MS through upregulation of the expression of CCR6 on circulating CD4+ $\mathrm{T}$ cells and induction in innate immune cells of the production of Th17 polarizing cytokines. Evidence is accumulating of a broader effect of airborne pollution on the incidence and progression of inflammatory and infectious disease, including an increased frequency and higher mortality of COVID-19 cases following increased exposure to air pollutants [30, 31]. Larger prospective studies are needed to confirm the effect of PM10 on Th17 cell polarization in MS patients, as well as to confirm the effect of PM10 on migratory properties of circulating lymphocyte and loss of BBB integrity. Future studies should also aim at understanding whether the pro-inflammatory effect of PM10 is MS specific or can also be observed across other autoimmune diseases or in physiological conditions. Also, it is not known whether the observed effect of PM10 depends mainly on its physical properties or on chemical properties of one or more of its components. Finally, molecular pathways underlying the cytokine-inducing activity of PM10 on DC are still largely unknown and will need to be elucidated.

\section{Abbreviations}

CCR6: C-C chemokine receptors 6; mdDC: Monocyte-derived dendritic cells; MS: Multiple sclerosis; PBMC: Blood mononuclear cells; PM10: Particulate matter 10; Th17: T helper 17 cells

\section{Acknowledgments}

Not applicable

\section{Authors' contributions}

Andrea Cortese, study concept and design, analysis and interpretation of data, draft of the manuscript. Luca Lova, acquisition and analysis of data. Patrizia Comoli, acquisition and analysis of data. Elisabetta Volpe, acquisition and analysis of data. Silvia Villa, acquisition, analysis and interpretation of data, draft of a portion of the manuscript. Giulia Mallucci, draft of a portion of the manuscript and critical revision of the manuscript. Sabrina La Salvia, acquisition and analysis of data. Alfredo Romani, study concept and design, analysis and interpretation of data, critical revision of the manuscript. Diego Franciotta, study concept and design, critical revision of the manuscript. Valentina Bollati, study concept, critical revision of the manuscript. Sabrina Basso, acquisition and analysis of data. Ilaria Guido, acquisition and analysis of data. Giuseppe Quartuccio, acquisition and analysis of data. Stella

Gagliardi, acquisition and analysis of data. Luca Battistini, acquisition and analysis of data, critical revision of the manuscript. Cristina Cereda, acquisition and analysis of data, critical revision of the manuscript. Roberto Bergamaschi study concept and design, study supervision, critical revision of the manuscript. The authors read and approved the final manuscript. 


\section{Funding}

A.C. thanks the Medical Research Council (MR/T001712/1), the Fondazione CARIPLO (2019-1836), and the Italian Ministry of Health Ricerca Corrente 2018-2019 for grant support.

\section{Availability of data and materials}

Anonymized data from this study will be shared by request from any qualified investigator.

\section{Ethics approval and consent to participate}

The Ethic Committee of San Raffaele Hospital, Milan, Italy, approved study protocol, and all subjects signed informed consent form.

\section{Consent for publication}

Not applicable

\section{Competing interests}

Andrea Cortese-reports no disclosures; Luca Lova—reports no disclosures ; Patrizia Comoli-reports no disclosures; Elisabetta Volpe-reports no disclosures; Silvia Villa-reports no disclosures; Giulia Mallucci-reports no disclosures; Sabrina La Salvia-reports no disclosures; Alfredo Romani-reports no disclosures; Diego Franciotta-reports no disclosures; Valentina Bollati-reports no disclosures; Sabrina Basso-reports no disclosures; llaria Guido—reports no disclosures; Giuseppe Quartuccio—reports no disclosures; Luca Battistini-reports no disclosures; Cristina Cereda-reports no disclosures; Roberto Bergamaschi—has served on scientific advisory boards for Biogen Idec and Almirall; has received funding for travel and speaker honoraria from Sanofi-Aventis, Genzyme, Biogen Idec, Bayer Schering, Teva Neurosciences, Merck Serono, Almirall, Roche and Novartis; has received research support from Merck Serono, Biogen Idec, Teva Neurosciences, Bayer Schering, Novartis, Sanofi-Aventis

\section{Author details}

${ }^{1}$ Department of Brain and Behavioural Sciences, University of Pavia, Pavia, Italy. ${ }^{2}$ Department of Neuromuscular Diseases, UCL Queen Square Institute of Neurology, Queen Square, London, UK. ${ }^{3}$ Biosciences S.p.A., Milan, Italy. ${ }^{4}$ RCCS Policlinico S. Matteo Foundation, Pavia, Italy. ${ }^{5}$ RCCS Santa Lucia Foundation, Rome, Italy. ${ }^{6}$ IRCCS Mondino Foundation, Pavia, Italy. ${ }^{7}$ University of Milan, Milan, Italy.

\section{Received: 5 May 2020 Accepted: 1 October 2020}

\section{Published online: 06 November 2020}

\section{References}

1. Mallucci G, Peruzzotti-Jametti L, Bernstock JD, Pluchino S. The role of immune cells, glia and neurons in white and gray matter pathology in multiple sclerosis. Prog Neurobiol. 2015;127-128:1-22.

2. Sospedra M, Martin R. Immunology of multiple sclerosis. Annu Rev Immunol. 2005;23:683-747.

3. Spelman T, Gray O, Trojano M, Petersen T, Izquierdo G, Lugaresi A, et al. Seasonal variation of relapse rate in multiple sclerosis is latitude dependent. Ann Neurol. 2014;76(6):880-90.

4. Fitzgerald KC, Munger KL, Kochert K, Arnason BG, Comi G, Cook S, et al. Association of vitamin $\mathrm{D}$ levels with multiple sclerosis activity and progression in patients receiving interferon beta-1b. JAMA Neurol. 2015; 72(12):1458-65.

5. Farez MF, Mascanfroni ID, Mendez-Huergo SP, Yeste A, Murugaiyan G, Garo $L P$, et al. Melatonin contributes to the seasonality of multiple sclerosis relapses. Cell. 2015;162(6):1338-52.

6. Saresella M, Mendozzi L, Rossi V, Mazzali F, Piancone F, LaRosa F, et al. Immunological and clinical effect of diet modulation of the gut microbiome in multiple sclerosis patients: a pilot study. Front Immunol. 2017;8:1391.

7. Tremlett H, Waubant E. The gut microbiota and pediatric multiple sclerosis: recent findings. Neurotherapeutics. 2018;15(1):102-8.

8. McKay KA, Jahanfar S, Duggan T, Tkachuk S, Tremlett H. Factors associated with onset, relapses or progression in multiple sclerosis: a systematic review. Neurotoxicology. 2017;61:189-212.

9. Bergamaschi R, Cortese A, Pichiecchio A, Berzolari FG, Borrelli P, Mallucci G, et al. Air pollution is associated to the multiple sclerosis inflammatory activity as measured by brain MRI. Mult Scler. 2018;24(12):1578-84.
10. Tateo F, Grassivaro F, Ermani M, Puthenparampil M, Gallo P. PM2.5 levels strongly associate with multiple sclerosis prevalence in the Province of Padua, Veneto Region, North-East Italy. Mult Scler. 2018;1352458518803273.

11. Angelici L, Piola M, Cavalleri T, Randi G, Cortini F, Bergamaschi R, et al. Effects of particulate matter exposure on multiple sclerosis hospital admission in Lombardy region, Italy. Environ Res. 2016;145:68-73.

12. Jeanjean M, Bind MA, Roux J, Ongagna JC, de Seze J, Bard D, et al. Ozone, NO2 and PM10 are associated with the occurrence of multiple sclerosis relapses. Evidence from seasonal multi-pollutant analyses. Environ Res. 2018;163:43-52.

13. Oikonen M, Laaksonen M, Laippala P, Oksaranta O, Lilius EM, Lindgren S, et al. Ambient air quality and occurrence of multiple sclerosis relapse. Neuroepidemiology. 2003;22(1):95-9.

14. Roux J, Bard D, Le Pabic E, Segala C, Reis J, Ongagna JC, et al. Air pollution by particulate matter PM10 may trigger multiple sclerosis relapses. Environ Res. 2017:156:404-10.

15. Comoli P, Basso S, Azzi A, Moretta A, De Santis R, Del Galdo F, et al. Dendritic cells pulsed with polyomavirus BK antigen induce ex vivo polyoma BK virusspecific cytotoxic T cell lines in seropositive healthy individuals and renal transplant recipients. J Am Soc Nephrol. 2003;14(12):3197-204.

16. Langrish $\mathrm{CL}$, Chen Y, Blumenschein WM, Mattson J, Basham B, Sedgwick JD, et al. IL-23 drives a pathogenic T cell population that induces autoimmune inflammation. J Exp Med. 2005;201(2):233-40.

17. McGeachy MJ, Bak-Jensen KS, Chen Y, Tato CM, Blumenschein W, McClanahan T, et al. TGF-beta and IL- 6 drive the production of IL-17 and IL10 by $T$ cells and restrain $T(H)-17$ cell-mediated pathology. Nat Immunol. 2007:8(12):1390-7.

18. Cohen AJ, Brauer M, Burnett R, Anderson HR, Frostad J, Estep K, et al. Estimates and 25-year trends of the global burden of disease attributable to ambient air pollution: an analysis of data from the Global Burden of Diseases Study 2015. Lancet. 2017;389(10082):1907-18.

19. Kelly FJ, Fussell JC. Linking ambient particulate matter pollution effects with oxidative biology and immune responses. Ann N Y Acad Sci. 2015;1340:84-94.

20. Sallusto F, Impellizzieri D, Basso C, Laroni A, Uccelli A, Lanzavecchia A, et al. Tcell trafficking in the central nervous system. Immunol Rev. 2012;248(1):216-27.

21. Reboldi A, Coisne C, Baumjohann D, Benvenuto F, Bottinelli D, Lira S, et al. $\mathrm{C}-\mathrm{C}$ chemokine receptor 6 -regulated entry of $\mathrm{TH}-17$ cells into the CNS through the choroid plexus is required for the initiation of EAE. Nat Immunol. 2009;10(5):514-23.

22. Miller $\mathrm{DH}$, Khan $\mathrm{OA}$, Sheremata $W A$, Blumhardt LD, Rice GP, Libonati MA, et al. A controlled trial of natalizumab for relapsing multiple sclerosis. $N$ Engl J Med. 2003;348(1):15-23.

23. Miossec P, Korn T, Kuchroo VK. Interleukin-17 and type 17 helper T cells. $N$ Engl J Med. 2009;361 (9):888-98.

24. Brucklacher-Waldert V, Stuerner K, Kolster M, Wolthausen J, Tolosa E. Phenotypical and functional characterization of T helper 17 cells in multiple sclerosis. Brain. 2009;132(Pt 12):3329-41.

25. Kish L, Hotte N, Kaplan GG, Vincent R, Tso R, Ganzle M, et al. Environmental particulate matter induces murine intestinal inflammatory responses and alters the gut microbiome. PLoS One. 2013;8(4):e62220.

26. Becker S, Soukup JM, Gilmour MI, Devlin RB. Stimulation of human and rat alveolar macrophages by urban air particulates: effects on oxidant radical generation and cytokine production. Toxicol Appl Pharmacol. 1996;141(2):637-48.

27. Quintana FJ, Basso AS, Iglesias AH, Korn T, Farez MF, Bettelli E, et al. Control of $T($ reg $)$ and $T(H) 17$ cell differentiation by the aryl hydrocarbon receptor. Nature. 2008:453(7191):65-71.

28. Matthews NC, Faith A, Pfeffer P, Lu H, Kelly FJ, Hawrylowicz CM, et al. Urban particulate matter suppresses priming of $\mathrm{T}$ helper type 1 cells by granulocyte/macrophage colony-stimulating factor-activated human dendritic cells. Am J Respir Cell Mol Biol. 2014;50(2):281-91.

29. Odoardi F, Sie C, Streyl K, Ulaganathan VK, Schlager C, Lodygin D, et al. T cells become licensed in the lung to enter the central nervous system. Nature. 2012;488(7413):675-9.

30. Zhao CN, Xu Z, Wu GC, Mao YM, Liu LN, et al. Emerging role of air pollution in autoimmune diseases. Autoimmun Rev. 2019;18(6):607-14.

31. Zhu Y, Xie J, Huang F, Cao L. Association between short-term exposure to air pollution and COVID-19 infection: evidence from China. Sci Total Environ. 2020;727:138704. https://doi.org/10.1016/j.scitotenv.2020.138704.

\section{Publisher's Note}

Springer Nature remains neutral with regard to jurisdictional claims in published maps and institutional affiliations. 